\title{
An Age-Structured Epidemic Model for Monitoring and Control of HIV/AIDS
}

\author{
Ping $\mathrm{Yan}^{1}$ and Teng $\mathrm{Lv}^{2, *}$ \\ ${ }^{1}$ School of Science, Anhui Agricultural University, Hefei 230036, China \\ ${ }^{2}$ School of Information Engineering, Anhui Xinhua University, Hefei 230088, China \\ *Corresponding author
}

\begin{abstract}
In order to interpret the importance of monitoring and control of HIV/AIDS, according to our ideas of monitoring and control of HIV/AIDS, a continuous SUI epidemic model for HIV/AIDS is established in which $S$ denotes susceptible individuals, $U$ denotes individuals infected with HIV but undiscovered and I denotes individuals discovered to be infected with HIV virus. Since chronological age and infection age play important roles in the infectivity of infected populations, it is significant to study HIV/AIDS models with chronological age and infection age.
\end{abstract}

Keywords-age-structure; SUI epidemic model; HIV/AIDS

\section{INTRODUCTION}

Epidemic models study the transmission dynamics of infectious disease in host populations, aiming at tracing factors that give rise to their occurrence. Sexually transmitted diseases such as HIV/AIDS(Human Immunodeficiency Virus/Acquired Immunodeficiency Syndrome), make more and more researchers develop suitable mathematical models and study their dynamic behaviors to describe and interpret the epidemiology of sexually transmitted infections.

HIV virus has a long incubation and infectious period (from 8 years to 10 years), and no vaccines for HIV virus are available till now. Many authors try their best to interpret the pathologic process of HIV/AIDS with mathematical models in recent years[1]-[11]. Since the infectivity of infected people varies according to their infection time and their chronological ages during the incubation period, a large number of agestructured mathematical models [1]-[9] have been developed to describe the population dynamics of HIV/AIDS.

Few models discussed the effects of monitoring and control of AIDS, in order to interpret the importance of monitoring and control of AIDS and according to the truth that no individuals can obtain the immunity to HIV/AIDS epidemic. Ignoring the recovered group, a continuous SUI epidemic model is established in which the population is divided into three groups: $\mathrm{S}$ (susceptible individuals uninfected with HIV virus but susceptible to them), U (individuals infected with HIV but undiscovered) and I (individuals with AIDS or with HIVpositive, that is, individuals discovered to be infected with HIV virus). Different from removed group in the mathematical sense, I-group contains no recovered individual and individuals in I-group are all patients who need medical treatments.
Since infection age also plays an important role in the infectivity of infected populations, it is significant to study dynamic behaviors of HIV/AIDS model. Unlike the models mentioned above, we introduce chronological age and infection age at the same time. In this paper, the continuous SUI epidemic model with chronological age and infection age is studied.

The rest of this paper is organized as follows. In Section 2, we establish a chronological-age and infection-age structured SUI continuous epidemic model for HIV/AIDS epidemic with partial differential equations. Some assumptions are also given in this section to assure the well-posedness of the model and the stability of the equilibrium solution. The existence and uniqueness of solution to the SUI model is discussed in Section 3. Section 4 is conclusions of our results.

\section{MODEL AND ASSUMPTIONS}

According to the truth that no individuals can obtain the immunity to HIV/AIDS epidemic and ignoring the recovered group, we divide the population into three epidemiologically distinct groups: S (susceptible individuals uninfected with HIV virus but susceptible to them), $U$ (individuals infected with HIV but undiscovered) and I (individuals with AIDS or with HIV-positive, that is, individuals discovered to be infected with HIV virus). Different from removed group in the mathematical sense, I-group contains no recovered individual and individuals in I-group are all patients who need medical treatments. We assume that individuals with HIV-positive in I-group can make sexual contacts with others by adopting protective measures and can not infect susceptible individuals. It is convenient to assume that individuals in U-group and I-group can have their health babies with the help of advanced technology of medicine and science.

Let $S=S(a, t)$ denote the age-density of susceptible population at time $t$ and chronological age $a$. Let $a_{m}$ be the highest age attained by the individuals in the host population. Let $U=U(a, \theta, t)$ be the density of undiscovered individuals infected with HIV virus at chronological age $a$, infection age $\theta$ and time $t$. Let $I=I(a, \theta, t)$ be the density of individuals with HIV-positive or AIDS at chronological age $a$, infection age $\theta$ and time $t$. For any given time $t \geq 0, U(a, \theta, t)$ and $I(a, \theta, t)$ are defined on the following domain 


$$
\Omega=\left\{(a, \theta) \mid 0<a<a_{m}, 0<\theta<a\right\} .
$$

The density of undiscovered individuals infected with HIV virus at chronological age $a$ and time $t$ can be given by

$$
\tilde{U}(a, t)=\int_{0}^{a} U(a, \theta, t) d \theta
$$

and the density of individuals with HIV-positive at chronological age $a$ and time $t$ can be given by

$$
\widetilde{I}(a, t)=\int_{0}^{a} I(a, \theta, t) d \theta
$$

Let $\mu(a)$ and $\gamma(a, \theta)$ denote the age-specific mortality rate, the rate of transition from U-group to I-group and the birth rate of the population, respectively.

Here, in order to interpret the importance of monitoring and control of AIDS and to discuss conveniently dynamic behaviors of the SUI model, we assume that all newborns belong to S-group, i.e., individuals in U-group and I-group can have their health babies with the help of advanced technology of medicine and science.

The assumption that individuals in I-group can adopt protective measures not to infect susceptible individuals when they make sexual contacts with others means that in our model only individuals in \$U\$-group can transmit the HIV virus by effectively contacting with susceptible individuals. According to the mechanism of infection, we introduce the following agespecific force of infection denoted by $\lambda(a, t)$ :

$$
\lambda(a, t)=\kappa_{1}(a) \int_{0}^{a_{m}} \int_{0}^{\sigma} \kappa_{2}(\sigma, \theta) U(\sigma, \theta, t) d \theta d \sigma,
$$

where $\kappa_{1}(a)$ and $\kappa_{2}(a, \theta)$ denote theage-specific contagion rate and the age-infection-specific infectious rate, respectively. They satisfy the following assumption.

(H0) $\kappa_{1}(a) \geq 0$ is continuous on $\left[0, a_{m}\right]$ and $\kappa_{2}(a, \theta) \geq 0$ is continuous on $\bar{\Omega}$.

Hence we have an initial boundary value problem as follows.

$$
\begin{gathered}
\frac{\partial S}{\partial t}+\frac{\partial S}{\partial a}=-(\mu(a)+\lambda(a, t)) S, \\
\frac{\partial U}{\partial t}+\frac{\partial U}{\partial a}+\frac{\partial U}{\partial \theta}=-(\mu(a)+\gamma(a, \theta)) U, \\
\frac{\partial I}{\partial t}+\frac{\partial I}{\partial a}+\frac{\partial I}{\partial \theta}=-\mu(a) I+\gamma(a, \theta) U,
\end{gathered}
$$

$$
S(a, 0)=S^{0}(a), U(a, \theta, 0)=U^{0}(a, \theta),
$$

$$
I(a, \theta, 0)=I^{0}(a, \theta),
$$

$$
S(0, t)=\int_{0}^{a_{m}} \beta(a)(S(a, t)+\tilde{U}(a, t)+\tilde{I}(a, t)) d a,
$$

$$
U(\theta, \theta, 0)=0, U(a, 0, t)=\lambda(a, t) S(a, t),
$$

$$
\mathrm{I}(\theta, \theta, 0)=0, \quad I(a, 0, t)=0 .
$$

In this model we suppose that the disease does not affect the death rate and the birth rate, so $\$ \backslash m u \$$ is taken to be the same for all three groups. We also make the following hypotheses: (H1) $\beta, \mu$ and $p_{0}$ are nonnegative, piecewise continuous functions on $\left[0, a_{m}\right)$.

(H2) $\beta(a)>0$ for $a \in\left(A_{0}, A_{1}\right)$ and $\beta(a)=0$ for $a \notin\left(A_{0}, A_{1}\right)$, where $A_{0}$ and $A_{1}$ are positive constants and satisfy $A_{0}<A_{1}<a_{m}$. The interval $\left(A_{0}, A_{1}\right)$ means reproduction period.

(H3) $\int_{0}^{a_{m}} \mu(\sigma) d \sigma<+\infty$ such that $\int_{0}^{a_{m}} e^{-\int_{0}^{a} \mu(\sigma) d \sigma} d a<+\infty$.

At the same time, we assume that the initial density of every group is nonnegative, which is biologically significant:

(H4) $S^{0}(a) \geq 0, U^{0}(a, \theta) \geq 0, I^{0}(a, \theta) \geq 0$.

\section{EXISTENCE AND UNIQUENESS OF THE SOLUTION TO SYSTEM (3)-(10)}

Let $\Gamma_{1}, \Gamma_{2}$ be nonnegative real numbers or $\infty$. Let the solution space be defined as follows.

$$
X\left(\Gamma_{1}, \Gamma_{2}\right)=X_{1}\left(\Gamma_{1}, \Gamma_{2}\right) \times X_{2}\left(\Gamma_{1}, \Gamma_{2}\right) \times X_{2}\left(\Gamma_{1}, \Gamma_{2}\right),
$$

where

$$
X_{1}\left(\Gamma_{1}, \Gamma_{2}\right)=C\left(\left[\Gamma_{1}, \Gamma_{2}\right] ; L^{1}\left(\left[0, a_{m}\right]\right)\right)
$$

and

$$
X_{2}\left(\Gamma_{1}, \Gamma_{2}\right)=C\left(\left[\Gamma_{1}, \Gamma_{2}\right] ; L^{1}(\Omega)\right)
$$

These spaces have the following norms:

$$
\begin{gathered}
\left\|f_{1}\right\|_{X_{1}\left(\Gamma_{1}, \Gamma_{2}\right)}=\sup _{\Gamma_{1} \leq t<\Gamma_{2}} \int_{0}^{a_{m}}\left|f_{1}(a, t)\right| d a, \\
\left\|f_{i}\right\|_{X_{1}\left(\Gamma_{1}, \Gamma_{2}\right)}=\sup _{\Gamma_{1} \leq t<\Gamma_{2}} \int_{0}^{a_{m}} \int_{0}^{a}\left|f_{1}(a, \theta, t)\right| d \theta d a, \quad i=2,3
\end{gathered}
$$


and

$$
\left\|\left(f_{1}, f_{2}, f_{3}\right)\right\|_{X\left(\Gamma_{1}, \Gamma_{2}\right)}=\sqrt{\left\|f_{1}\right\|_{X_{1}\left(\Gamma_{1}, \Gamma_{2}\right)}^{2}+\left\|f_{2}\right\|_{X_{2}\left(\Gamma_{1}, \Gamma_{2}\right)}^{2}+\left\|f_{3}\right\|_{X_{2}\left(\Gamma_{1}, \Gamma_{2}\right)}^{2}} .
$$

In order to discuss conveniently the existence and uniqueness of the solution to system (3)-(10), we introduce the following function transformation

$$
\begin{gathered}
s(a, t)=\frac{S(a, t)}{p_{\infty}(a)}, \\
u(a, \theta, t)=\frac{U(a, \theta, t)}{p_{\infty}(a)}, \\
i(a, \theta, t)=\frac{I(a, \theta, t)}{p_{\infty}(a)},
\end{gathered}
$$

where

$$
p_{\infty}(a)=b_{0} e^{\int_{0}^{a} \mu(\sigma) d \sigma}
$$

and

$$
b_{0}=\int_{0}^{a_{m}} p_{\infty}(a) d a
$$

Then system (\ref\{11\})-(\ref\{110\}) can be changed into

$$
\begin{gathered}
\frac{\partial s}{\partial t}+\frac{\partial s}{\partial a}=-\lambda(a, t) s(a, t), \\
\frac{\partial u}{\partial t}+\frac{\partial u}{\partial a}+\frac{\partial u}{\partial \theta}=-\gamma(a, \theta) u(a, \theta, t), \\
\frac{\partial i}{\partial t}+\frac{\partial i}{\partial a}+\frac{\partial i}{\partial \theta}=\gamma(a, \theta) u(a, \theta, t), \\
s(a, 0)=s^{0}(a), u(a, \theta, 0)=u^{0}(a, \theta), i(a, \theta, 0)=i^{0}(a, \theta), \\
s(0, t)=1, u(\theta, \theta, 0)=0, u(a, 0, t)=\lambda(a, t) s(a, t), \\
i(\theta, \theta, 0)=0, \quad i(a, 0, t)=0 .
\end{gathered}
$$

where

$$
\lambda(a, t)=\kappa_{1}(a) \int_{0}^{a_{m}} \int_{0}^{\sigma} \kappa_{2}(\sigma, \theta) p_{\infty}(\sigma) u(\sigma, \theta, t) d \theta d \sigma,
$$

$$
\begin{gathered}
s^{0}(a)=\frac{S^{0}(a)}{p_{\infty}(a)}, \\
u^{0}(a, \theta)=\frac{U^{0}(a, \theta)}{p_{\infty}(a)}, \\
i^{0}(a, \theta)=\frac{I^{0}(a, \theta)}{p_{\infty}(a)}, \\
\tilde{u}(a, t)=\int_{0}^{a} u(a, \theta, t) d \theta
\end{gathered}
$$

and

$$
\tilde{i}(a, t)=\int_{0}^{a} i(a, \theta, t) d \theta
$$

Because of the reversibility of the function transformation (11), system (12)-(17) and system (12)-(17) are equivalent. It is sufficient to discuss the existence and the existence and uniqueness and stability of solutions to system (12)-(17). Using the method of characteristic lines in [12], system (12)-(17) admits a unique solution as given in the following theorem.

Theorem 3.1 If assumptions (H0)-(H4) hold, there exists a unique nonnegative solution $(s(a, t), u(a, \theta, t), i(a, \theta, t))$ in the solution space $X(0,+\infty)$ for system (12)-(17), where the unique solution $(s(a, t), u(a, \theta, t), i(a, \theta, t))$ depends on the initial data continuously andsatisfies the following integral system.

$$
\begin{aligned}
& s(a, t)= \begin{cases}s^{0}(a-t) e^{-\int_{0}^{t} \lambda(\tau+a-t, \tau) d \tau}, & a \geq t \\
e^{-\int_{t-a}^{t} \lambda(\tau+a-t, \tau) d \tau}, & a<t\end{cases} \\
& u(a, \theta, t)=\left\{\begin{array}{cc}
u^{0}(a-t, \theta-t) e^{-\int_{0}^{t} \gamma(\tau+a-t, \tau+\theta-t) d \tau}, & a \geq t, \theta \geq t \\
\lambda(a-\theta, t-\theta) s(a-\theta, t-\theta) e^{-\int_{0}^{\theta} \lambda(\tau+a-t, \tau) d \tau}, & a>\theta, t>\theta
\end{array}\right. \\
& i(a, \theta, t)=\left\{\begin{array}{rr}
i^{0}(a-t, \theta-t)+\int_{0}^{t} \gamma(\tau+a-t, \tau+\theta-t) u(\tau+a-t, \tau+\theta-t) d \tau \\
\quad a \geq t, \theta \geq t \\
\lambda(a-\theta, t-\theta) s(a-\theta, t-\theta)-u(a, \theta, t), \quad a>\theta, t>\theta
\end{array}\right.
\end{aligned}
$$

\section{CONCLUSIONS}


In order to interpret the importance of monitoring and control of AIDS, we assumed that individuals in U-group and I-group could have their babies with the help of advanced technology of medicine and science. This means that the route of the vertical transmission of HIV/AIDS diseases can be cut off by artificial interference. We assumed that individuals with HIV-positive in I-group could make sexual contacts with others by adopting protective measures and do not infect susceptible individuals. According to the practical significance, we talked about the case that only individuals in U-group could infect susceptible individuals by effectively contacting with each other. It was completely different from the models known before.

The infectivity of infected individuals varies depending on their infection age and their chronological age. Due to the important role of the infection age and the chronological age in the progress of HIV/AIDS epidemic, we introduced chronological age and infection age at the same time into both U-group and I-group. It was helpful to accurately analyze the transmission of HIV/AIDS epidemic.

For an infection-age-structured model of HIV/AIDS, our model ignored vertical transmission and assumed that individuals in three group had the same birth rate \$beta(a)\$. A more reasonable model of partial differential equations with vertical transmission will be discussed in future. Furthermore, we supposed that total population reached its equilibrium distribution because we assumed that the disease did not affect the death rate and the birth rate. In the future work, we will discuss the case in which the individuals in \$I-group will die because of the disease.

\section{ACKNOWLEDGMENT}

This research was supported by National Nature Science Foundation of China (No.11201002), Anhui Province Colleges Nature Science Research Project(No.KJ2015A325), Anhui Province Quality Engineering (2015zy073), Introduction of Talents Foundation and Academic Leader Foundation of Anhui Xinhua University (2014XXK06). .

\section{REFERENCES}

[1] S. Busenberg and C. Castillo-Chavez, "A general solution of the problem of mixing of subpopulations and its application to risk- and agestructured models for the spread of AIDS,” IMA J. Math. Appl. Med. Biol., Vol.8, pp. 1-29, 1991.

[2] L.M. Cai, X.Z. Li, M. Ghosh and B.Z. Guo, "Stability analysis of an HIV/AIDS epidemic model with treatment," J. Comp. and Appl. Math., vol.229, pp.313-323, 2009.

[3] X.Z. Li, J.X. Liu and M. Martcheva, "An age-structured two-strain epidemic model with super-infection," Math. Biosci. Eng., vol.7, pp. 123-147, 2010.

[4] M. Martcheva and X.Z. Li, "Linking immunological and epidemiological dynamics of HIV: the case of super-infection,” J. Biol . Dyn., vol. 7, pp. 161-182, 2013.

[5] J.M. Heffernan, R.J. Smith and L.M. Wahl, "Perspectives on the basic reproductive ratio,” J. R. Soc. Interface., vol.2, pp. 281-283, 2005.

[6] J.M. Hyman, J. Li and E.A. Stanley, "Threshold conditions for the spread of the HIV infection in an age-structured populations of homosexual men,” J. Theor. Biol., vol.166, pp. 9-31, 1994.
[7] H. Inaba, "Endemic threshold results in an age-duration-structured population model for HIV infection,”. Math. Biosci., vol. 201, pp. 15-47, 2006.

[8] R.M. May and R.M. Anderson, "Transmission dynamics of HIV infection,” Nature, Vol. 326, pp.137-142, 1987.

[9] R.M. May, M. Anderson and A.R. McLean, "Possible demographic consequences of HIV/AIDS,” Math. Biosci., vol. 90, pp. 475-506, 1988.

[10] A.S. Perelson, "Modelling viral and immune system dynamics," Nat. Rev.: Immunal, vol.2, pp. 28-36, 2002.

[11] D. Wodarz, K. Page, R. Arnaout, A.R. Thomsen, J.D. Lifson and M.A Nowak, "A new theory of cytotoxic T-lymphocyte memory: implications for HIV treatment. Philos,” Trans. R. Soc. Lond., vol. B355 pp. 329-343, 2000.

[12] P. Yan, "Wellposedness of the model for the dynamics of infectious diseases without immunity and with latent period(in Chinese),”. J.. Biomath., vol. 23, pp. 245-256, 2008. 